\title{
Fixed-target serial synchrotron crystallography combined with HAG method for room temperature measurement
}

Takashi Kumasaka ${ }^{a}$, Kazuya Hasegawa ${ }^{a}$, Seiki Baba ${ }^{a}$, Takashi Kawamura ${ }^{a}$, Keitaro Yamashita $^{2}$, Kunio Hirata ${ }^{3}$, Masaki Yamamoto ${ }^{3}$

aJapan Synchrotron Radiation Research Institute, 1-1-1 Kouto, Sayo 6795198, Japan, kumasaka@spring8.or.jp

${ }^{\mathrm{b}}$ The University of Tokyo

${ }^{\mathrm{C}}$ RIKEN SPring-8 Center

The serial femtosecond crystallography (SFX) developed at XFELs opened a new paradigm for MX data collection and successfully applied to the time-resolved (TR) analysis. Its success leads to establish synchrotron serial crystallography (SSX) by combining a high-flux microbeam and a fast-readout detector. And then SSX is going to evolve to TR-SSX, also called as serial millisecond crystallography (SMX). Most SMX methods are almost compatible to TR-SFX by using apparatuses developed at XFELs. It is useful to examine both methods and light sources, but some high barriers remain in approach from the conventional MX method.

As an alternate way to TR analysis, we propose to apply a capillary free mount technique, which can apply various experimental conditions, to SMX. It's our improved method, HAG (humid air and glue-coating) method, has more suitable features for room temperature SMX: it maintains most samples, which are fixated to cryoloops by glue [1]. It has already revealed the dynamical motions of H-Ras [2], cytochrome c oxidase [3], copper amine oxidase [4], and so on. We are now combining it to SS-ROX, our improved SSX [5], as a fixed-target SMX. This development is being conducted at SPring-8 BL41XU, where the pink beam will be available in future.

Conclusively, we have been totally engaged in the development of serial crystallography by using both SPring-8 micro-focus beamlines and XFEL SACLA.

\section{References}

[1] Baba et al., Acta Cryst D69, 1839 (2013)

[2] Matsumoto et al., Sci Rep 6, 25931 (2016)

[3] Shimada et al., Sci Adv 3, e1603042 (2017)

[4] Murakawa et al., PNAS, 116, 135 (2019)

[5] Hasegawa et al., J Sync Rad 24, 29 (2017) 